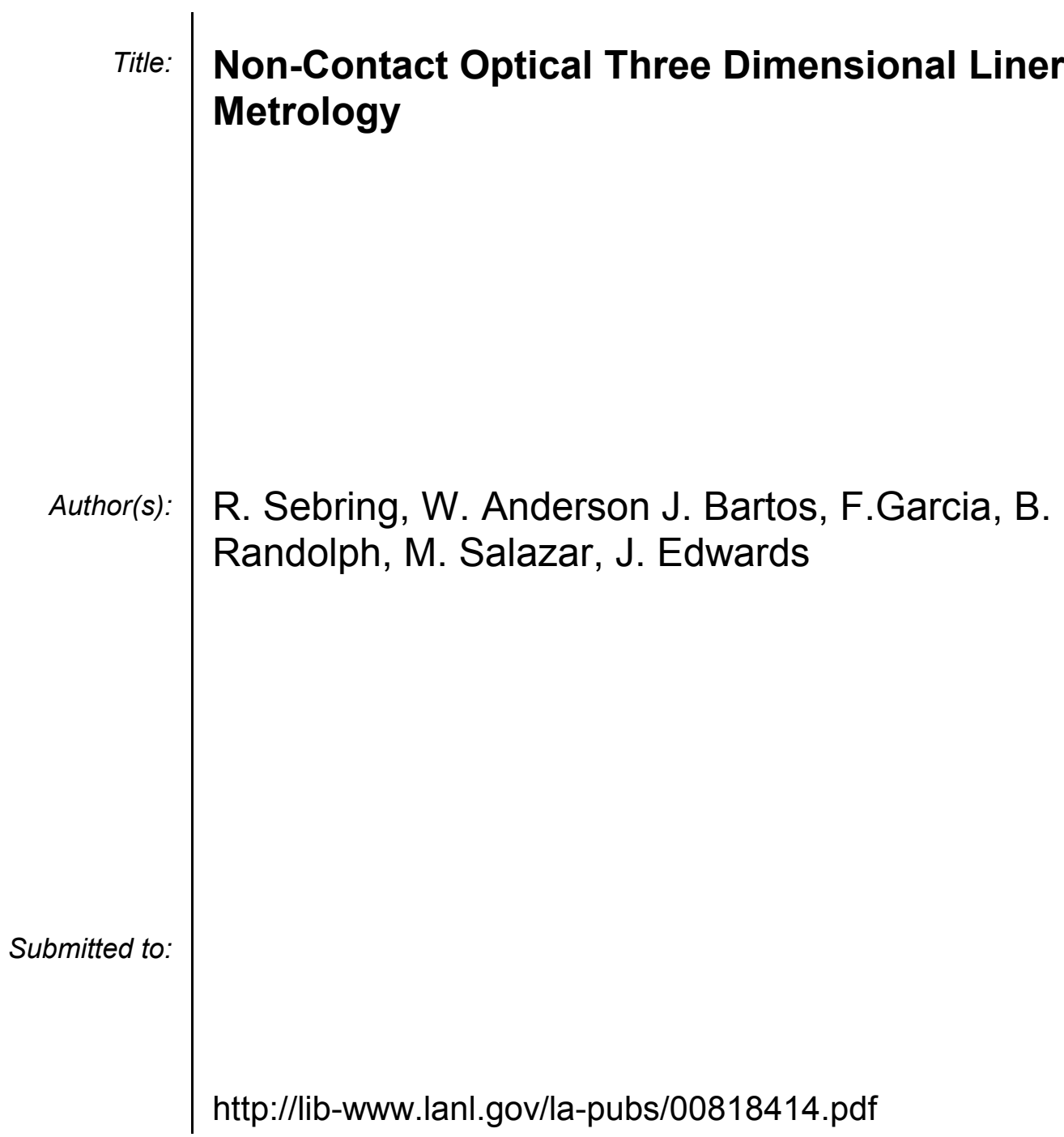

Los Alamos National Laboratory, an affirmative action/equal opportunity employer, is operated by the University of California for the U.S. Department of Energy under contract W-7405-ENG-36. By acceptance of this article, the publisher recognizes that the U.S. Government retains a nonexclusive, royaltyfree license to publish or reproduce the published form of this contribution, or to allow others to do so, for U.S. Government purposes. Los Alamos National Laboratory requests that the publisher identify this article as work performed under the auspices of the U.S. Department of Energy. Los Alamos National Laboratory strongly supports academic freedom and a researcher's right to publish; as an institution, however, the Laboratory does not endorse the viewpoint of a publication or guarantee its technical correctness. 


\title{
NON-CONTACT OPTICAL THREE DIMENSIONAL LINER METROLOGY
}

\author{
R. Sebring, W. Anderson J. Bartos, F.Garcia, B. Randolph, M. Salazar, J. Edwards \\ Los Alamos National Laboratory, MST-7, PO Box 1663, MS E-549 \\ Los Alamos, NM, 87545, USA
}

\begin{abstract}
We optically captured the "as-built" liner geometry of NTLX (near term liner experiments) for Shiva Star using ultra-precision ranging lasers. We subsequently verified the resulting digitized geometry against the $3 \mathrm{D}$ CAD model of the part. The results confirmed that the Liner contours are within designed tolerances but revealed subtle fabrication artifacts that would typically go undetected. These features included centimeters long waviness and saddle and bulge regions of 1 micron or less in magnitude. The laser technology typically provided 10 micron spatial resolution with \pm 12 nanometer ranging precision. Atlas liners in the future may have to be diamond turned and will have the centimeter wavelength and 100 angstrom amplitude requirements. The advantages of using laser technology are 1) it avoids surface damage that may occur with conventional contact probes and 2) dramatically improves spatial resolution over CMM, capacitance and inductance type probes. Our work is the result of a perceived future need to develop precision, non-contact, liner inspection techniques to verify geometry, characterize machining artifacts and map wall thickness on delicate diamond turned surfaces. Capturing "as-built" geometry in a non-contact way coupled with part-to-CAD verification software tools creates a new metrology competency for MST-7.
\end{abstract}

\section{INTRODUCTION}

Characterization of wall thickness variations is becoming an important issue in the fabrication of precision liners for the Los Alamos High Energy Density Hydrodynamics (HEDH) experiments. Presently our liners are inspected in a number of dimensional aspects including surface finish, length, diameter, straightness and nominal wall thickness. These measurements are typically one dimensional and can only describe a particular liner in a limited fashion. However, if one wishes to address variations in surface contour along a particular part axis or characterize how wall thickness varies spatially around the entire liner this becomes a three dimensional measurement problem. The implementation of a 3D metrology "mapping" strategy represents challenges for us primarily in three technical areas: (1) ultra-precision motion control and data acquisition (2) integration of suitable optical ranging probes to detect dimensional variations and (3) application of computer analytical verification software tools to make sense of the copious amounts of data. We developed a prototype optical measuring device to map the wall variations of a test liner and used advanced reverse engineering software to analyze the data. In this paper we report the experimental results of measuring the wall thickness variations on cylinders.

\section{MEASUREMENT PROCEDURE}

The cylindrical parts that were measured were in varying states of fabrication which required different setups of the part relative to the optical probe(s) and motions devices.

\section{A. Liner In Glide Plane Assembly}

This liner was assembled with two copper glide planes (Fig.1). The assembly remained stationary while the single laser probe was moved along the long axis of the cylinder using an ultra-precision mechanical stage (Newport, Model PM500-L). A single line scan was captured along the long axis of the liner on the outside surface only. The glide planes blocked access to the liner inside surface. The laser probe analog voltage data was acquired using a digital storage oscilloscope (HewlettPackard). The $\mathrm{X}$ axis position was estimated based upon known linear axis motion speed.

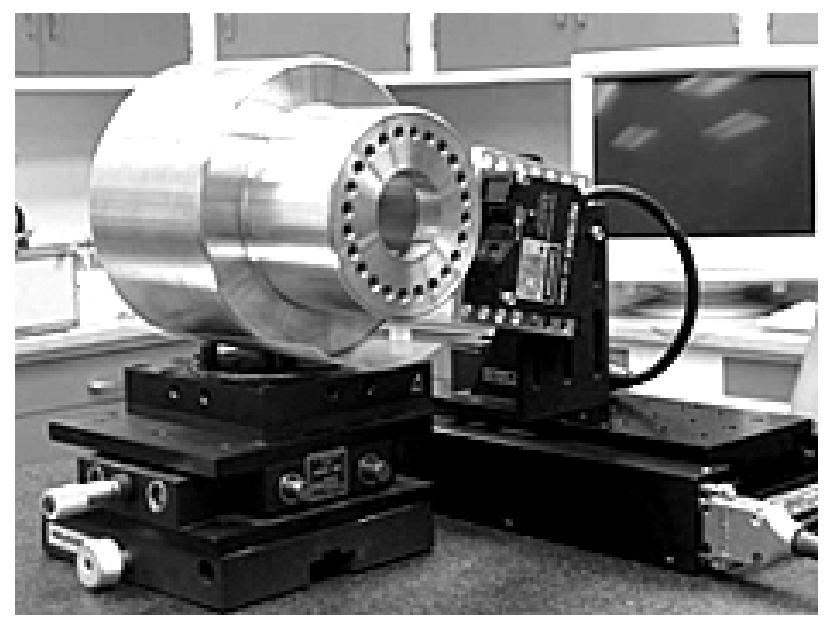

Figure 1. Measuring configuration for liner glide plane assembly with single laser probe to capture OD contour.

\section{B. Free-Standing Liner}

The second configuration was optimized to acquire single line contours from the inside (ID) and outside surfaces (OD) simultaneously on the liner (Fig.2) This 
allowed measurement of relative wall thickness variations as well as ID and OD contours. The Shiva Star liner had been incorporated into the glide plane assembly described above and was subsequently removed from the assembly. The free-standing liner permitted insertion of a laser probe inside the cylinder to contour the inside wall surface as well as the outside simultaneously.

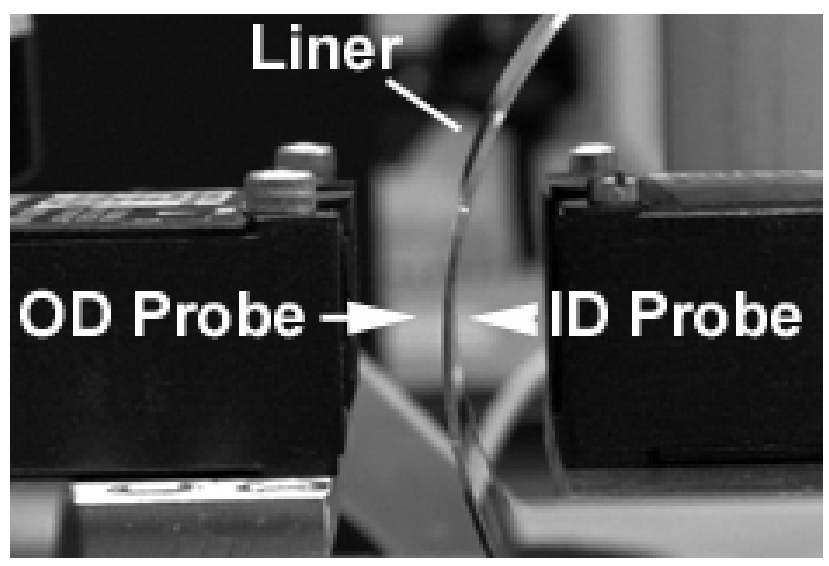

Figure 2. Measuring configuration for free standing Shiva Star liner with two opposing laser probes to capture ID and OD contour and wall thickness variations.

The two laser probes were mounted facing each other with the liner wall between them such that the laser angle was parallel to the cylindrical axis (Fig. 2). The liner remained stationary while both probes traveled along the entire cylinder length together on a single precision air bearing linear motion stage. Analog voltage probe data was recorded using the HP oscilloscope as before. $\mathrm{X}$ axis position relative to laser probe data was again estimated based upon known linear motion speed.

The liner and probes were mounted on our custom experimental measuring platform (EMP). The EMP (Fig. 3) consists of an $X$ axis, $Y$ axis and a rotary $W$ axis parallel to the $\mathrm{XY}$ plane. The $\mathrm{X}$ and $\mathrm{Y}$ are graphite air bearings on round ways mounted in a custom set of frames. Positioning is by linear motors. Rotary $\mathrm{W}$ is a 4 inch blockhead spindle (Precision Instruments). The XY resolution is 100 nanometer, repeatability is \pm 100 nanometers and $\mathrm{W}$ (rotary axis) resolution is .09 degrees, repeatability is \pm .09 degrees. The man/machine interface is LabView graphical programming software with Active $\mathrm{X}$ objects. Laser probes are solid state laser displacement detectors (Keyence Corp. Model LC-2420). Data acquisition is via quadrature counters operating at 8 megahertz, and 16 bit A to D converters writing to a PC hard drive.

\section{Mapping Aluminum Test Cylinder}

To fully characterize wall thickness variations over the entire cylindrical surface required us to rotate the part around its cylindrical axis while collecting wall contour (ID and OD) and thickness data as a function of rotation and lateral position. This can be visualized as making a thread cutting motion. We designed and fabricated a special test liner to help us develop our techniques in motion control, data acquisition, data analysis and to demonstrate the utility of reverse engineering a known cylindrical shape. The test cylinder was made from 1100 series aluminum. The rough cylinder shape was turned on a slant-bed lathe (Hardinge) and the final diamond turning on a $\mathrm{T}$ based lathe (Pneumo) using a single crystal diamond tool. The cylinder was mounted on the EMP (Fig. 3). The two ranging lasers were mounted in opposing configuration as with the free-standing liner above. Rotation and linear motion positions were recorded simultaneously with the analog output of the laser probes into a single text data file on a PC.

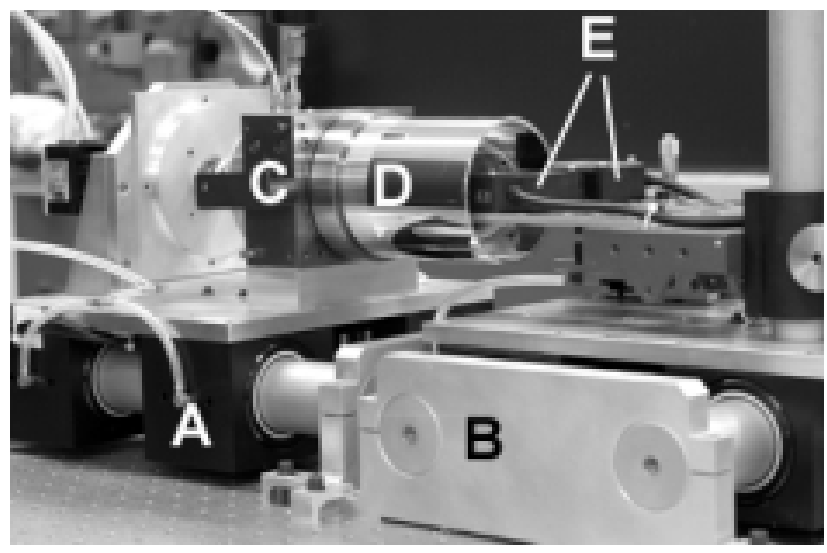

Figure 3. Experimental Measuring Platform (EMP) with linear $\mathrm{X}$ axis $(\mathrm{A})$, linear $\mathrm{Y}$ axis $(\mathrm{B})$, rotary $\mathrm{W}$ axis $(\mathrm{C})$, diamond turned aluminum cylinder (D), two opposing laser displacement sensors (E).

\section{Data Processing and Analysis}

Raw data from the motion axis and the laser probes were transformed into appropriate $\mathrm{YZ}$ and $\mathrm{XYZ}$ coordinate units using Excel spreadsheet software. The two dimensional (2D) YZ data from the Shiva Star liners were plotted in Excel. The three dimensional (3D) XYZ data from the test aluminum cylinder was imported into a reverse engineering and point surfacing software (SDRC, Freeform) where the points were verified against a nominal 3D CAD model of the part.

\section{RESULTS}

A single scan line contour (Fig. 4) on a portion of the liner in the glide plane assembly (Fig. 1) revealed a slightly concave profile with a waviness at periods of $0.120,3.3$ and $8.2 \mathrm{~mm}$. (Fig. 5). The total wall variation was within $\pm 0.5 \mu \mathrm{m}$. This was within manufacturing specification.

A dual laser displacement line scan (Fig. 6) on a free standing Shiva Star liner revealed that the wall was thicker near the central region by as much as $40 \mu \mathrm{m}$. and that the wall was generally thicker at one end than the 
other by about $20 \mu \mathrm{m}$ Relative ID and OD wall contours showed that most of this wall variation was due to the inside contour. This particular liner had been prviously assembled with the glide planes and then removed.

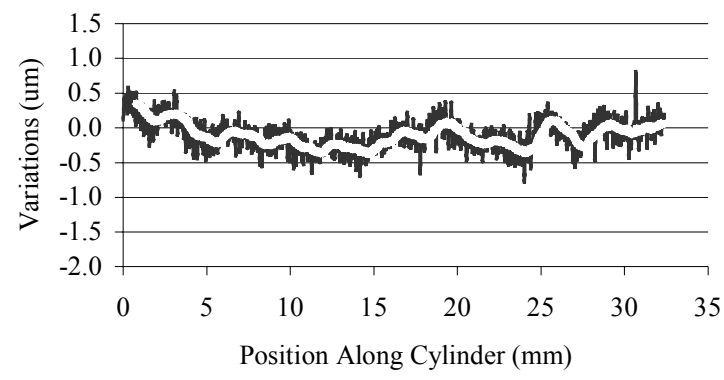

Figure 4. Contour of OD on Shiva Star liner in glide plane assembly.

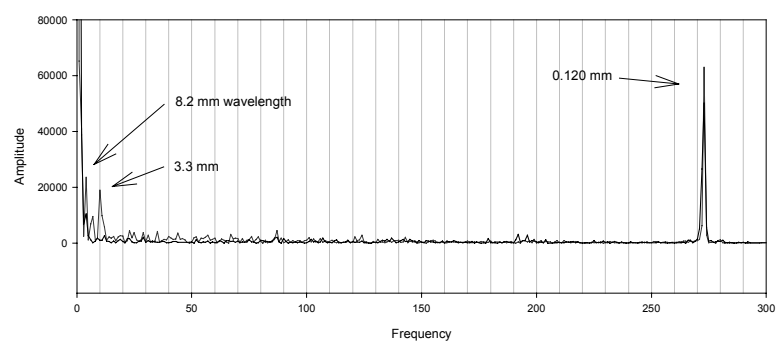

Figure 5. Power spectrum plot of data in Fig. 4 showing wavelength and relative magnitude of contour waviness.

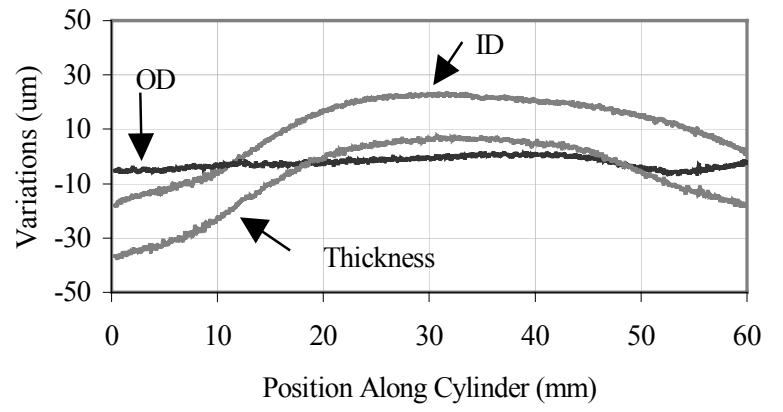

Figure 6. ID, OD and wall thickness variations of Shiva Star liner showing ID contour contributing majority of variation.

Wall thickness variations of the test aluminum cylinder provided a very dense data set of wall thickness information over the entire part (Fig.7). The point spacing around the approximately $320 \mathrm{~mm}$ circumference was 1 $\mathrm{mm}$ with helical period of $0.1 \mathrm{~mm}$. This provided a three dimensional point cloud in excess 230,000 coordinate data points. The point cloud was compared to a nominal CAD cylinder surface. The resulting verification of points-to-CAD differences revealed a roughly conical shape of the part. The brightly shaded regions are due to a large number of points outside the CAD surface and the darkly shaded regions are points lying on the inside relative to the CAD surface. Wall thickness artifacts formed fine rings around the circumference The thinner bright and dark bands are due to fine scratches on the ID surface of the cylinder. 


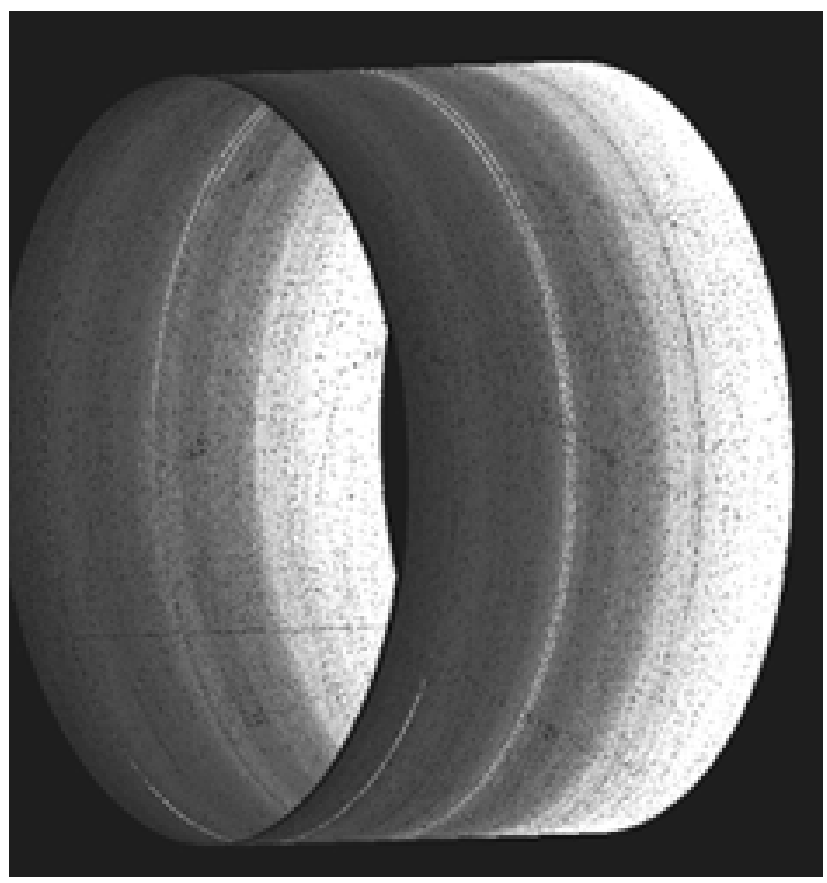

Figure 7. Cloud-To-CAD verification tolerance map showing conical shape and azmuthally varying wall thickness artifacts.

Fine artifacts may be better visualized by a needle plot where the needle length is proportional to the magnitude of the error (Figure 8). The needles have been enlarged one thousand time for visualization purposes.

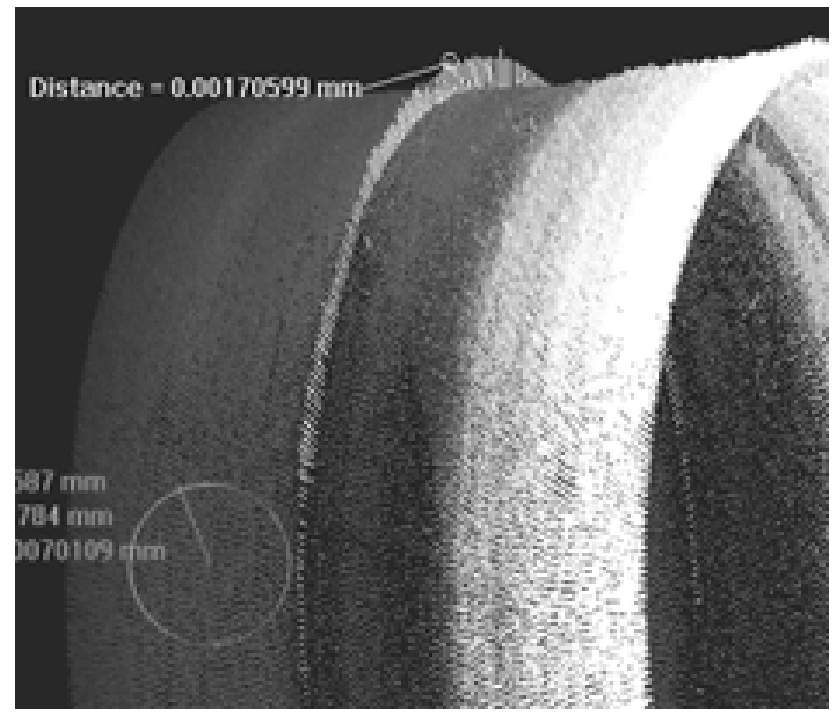

Figure 8. Cloud-To-CAD verification showing error needles proportional to distance of point from surface showing fine wall thickness variations due to scratches.

One of the longest needles in the image is only 0.0017 $\mathrm{mm}$ (1.7 microns) high and the circled region shows an averaged error of negative $0.0007 \mathrm{~mm}$ ( 0.7 microns). The error statistics for all the points showed the average error was $\pm 0.00018 \mathrm{~mm}$ with a standard deviation of \pm 0.00033 $\mathrm{mm}$. Statistical analysis of the as-built wall thickness deviations from nominal showed that $99.6 \%$ of the errors fell within $\pm 0.002 \mathrm{~mm}$ with an average of $\pm 0.0008 \mathrm{~mm}$ and \pm 0.0005 standard deviation.

The cylinder errors may be visualized and analyzed in a number of ways best suited for the wall thickness artifacts of interest. We found it useful to convert the data to a plane, virtually unrolling the cylinder and comparing the wall thickness variation to a best fit plane. The needle plots are again highly exaggerated by several thousand times the actual point to surface error. Note the four apparent rows in Figure 9. These are sub-micron wall thickness variations that represent a subtle wave not 
apparent in the cylindrical representation. The scratches also show up dramatically in this representation.

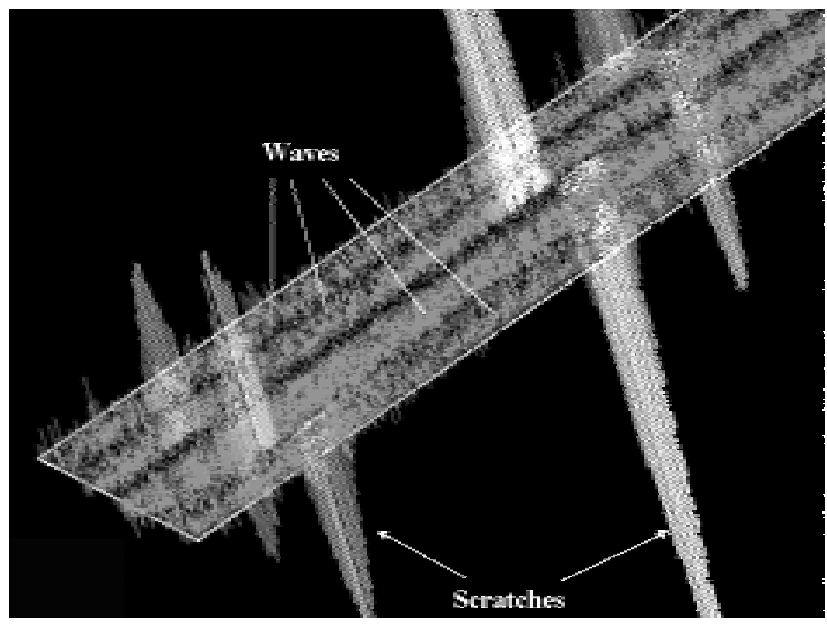

Figure 9. Unwrapped cylinder into planar view with verification of Cloud-To-CAD showing sub-micron waviness wall thickness variations and scratch type artifacts.

\section{DISCUSSION AND CONCLUSIONS}

Within the past year we have made significant strides in our optical mapping technology and methods. We have added a finer motion and more axes, true direct computer control of motion, integrated multiple streams of data acquisition, implemented a new man/machine software interface, acquired additional ultra-precision optical probes, and developed methods in data processing and three dimensional analytical and visualization techniques.

We present in this paper our results that cover a range from earlier simple wall contouring using a single probe on a single mechanical axis motion through continuous wall thickness variations using simultaneous dual probes on a three axis air bearing motion system that dimensionally maps and reverse engineers the entire cylindrical part.

For the Pegasus liners we used 400 data points from a coordinate measuring machine (CMM) in touch probe mode to asses the geometry of the as built HEDH liners. For higher surface finish requirements for Atlas liners, extreme care must be taken to avoid damage to the soft aluminum finish. Contact probe contouring such as with traditional CMM methods or even LVDT probes will be discouraged. Consequently, without metrology of contours, a great deal of faith must reside in the knowledge of the machining process itself. The liners are continually becoming more precise and our machining processes have not been adequately characterized at this greater level of precision. HEDH experiments in accelerating aluminum liners using pulsed power on Pegasus have confirmed code predictions as to how subtle variation or waviness in liner thickness may cause Rayleigh-Taylor instabilities [1]. Our laboratory demonstrated $80 \mu \mathrm{m}$ waviness on aluminum liner contours using an LVDT profiling device [2].

Our preliminary work, as presented in this paper, is a direct result of a need to map wall thickness variation over the entire region of interest of liners in a non-contact fashion. We have demonstrated that contour information on the outside wall alone does not necessarily represent what is happening on the inside wall. For example, localized artifacts around the axis of rotation of the part may not be detected by simple linear profiles in any one particular scan direction. This illustrates the need to map as much of a liner wall thickness as possible to better understand what is going on in the machining processes as well as verify what we actually fabricated. Because of the enormous amounts of coordinate data required for this type of mapping and the delicacy of the cylinder surface it will be necessary to use high speed non-contact probes, specialized precision motions, digital data collection and software for three dimensional model verification schemes.

\section{REFERENCES}

[1] Maurice Sheppard, Walter Atchison, Rhon Keinigs and John Stokes, "Rayleigh-Taylor Instability Growth Enigma: Liner Studies on Pegasus". in Proc. $12^{\text {th }}$ IEEE International Pulse Power Conference, 1999, p. 892.

[2] W. E. Anderson, J. J. Bartos, R. D. Day, F. P. Garcia, D. J. Hatch, R. B. Randolph and J. Townsend, "Fabrication of An Ultra Smooth Liner For Ranchero," in Proc. $12^{\text {th }}$ IEEE International Pulsed Power Conference, Vol. II, Monterey, California, June, 1999, p. 884. 